\title{
Why should Neurointervention be indexed in International Databases?
}

\author{
Sun Huh, MD
}

Neurointervention, the official journal of the Korean Society of Interventional Neuroradiology, publishes original articles documenting interventional neuroradiology biannually. It was launched in 2006. Beginning in 2011, it began to be published only in English. Therefore, beginning in 2011, it began building the foundation for becoming an invaluable international journal in the field of neurointervention. According to the NLM Catalog of journals referenced in the NCBI Databases, the following two journals are listed under neurointervention [1]:

Journal of neurointerventional surgery by the BMJ Publishing Group from 2009

Interventional neuroradiology by Edizioni del Centauro from 1995

However, in PubMed Central (PMC), there is no journal listed for the field. Neurointervention deals with a very specific field in neuroradiology and neurosurgery. Since currently most of the authors are from Korea, the journal content reflects the current rapid development of interventional neuroradiology techniques and neurointerventional surgery in Korea.

Department of Parasitology, College of Medicine and Institute of Medical Education, Hallym University, Chuncheon, Korea

Received June 1, 2011;

accepted after revision July 5, 2011.

Correspondence to: Sun Huh, MD, Department of Parasitology, College of Medicine, and Institute of Medical Education, Hallym University, 39 Hallym University Road, Chuncheon 200-702, Korea.

Tel. 82.33.248.2652 Fax. 82.33.241.1672

E-mail: shuh@hallym.ac.kr

This is an Open Access article distributed under the terms of the Creative Commons Attribution Non-Commercial License (http://creativecommons.org/licenses/by-nc/3.0) which permits unrestricted non-commercial use, distribution, and reproduction in any medium, provided the original work is properly cited.
There is a realistic possibility that papers could be cited and used by every specialist worldwide in this very specific field. It is a time for Neurointervention to become a PubMed Central (PMC) journal. It is not difficult for medical journals in English to be indexed in PMC if PMC XML files with the required quality are submitted. In 2007 the Korean Association of Medical Journal Editors (KAMJE) began offering the service of generating high quality PMC XML files of English-language journals from Korea to submit to PMC. PMC XML files are also used as the landing pages for digital object identifiers (DOIs) in KoreaMed Synapse, a digital archive and reference linking platform of Korean medical journals [2]. Every journal that is a member of the Korean Association of Medical Journal Editors can benefit by this service.

If a journal is indexed in PMC, it will be added to PubMed consequently. A variety of benefits result from becoming a PubMed journal. First, the citation frequency increases. Although in the first day of June 2011, only four articles from Neurointervention were cited by SCIE journals, the number of citations will rapidly increase when it is indexed in PubMed. Second, co-author networks through BioMedExperts can be easily developed [3]. Furthermore, when Neurointervention is indexed to the Web of Science or the SCOPUS database, there will be another chance to access the broader citation network of each paper and each author beyond that of Neurointervention. SCImagoJR provides the co-citation networks and hindex based on SCOPUS [4]. Beyond all this, most importantly, a listing in PubMed/PMC would provide high quality specialized information to clinicians, researchers, and laypeople worldwide without access fees. Since the quality of neurointerventional service in Korea is one of the best in the world, the medical information through this open access journal is invalu- 
able to them.

Just as the proverb says 'A good beginning is half the battle', Neurointervention is already poised leap to the status of an international journal. If every member of the Korean Society of Interventional Neuroradiology does his or her best to increase the journal's quality, the journal will be able to arrive at this goal very soon.

\section{References}

1. NLM Catalog: Journals referenced in the NCBI Databases [Internet]. Bethesda: U.S. National Library of Medicine; [cited
2011 Jun 1]. Available from: http://www.ncbi.nlm.nih.gov/ nlmcatalog/journals.

2. KoreaMed Synapse [Internet]. Seoul: Korean Association of Medical Journal Editors; [cited 2011 Jun 1]. Available from: http://synapse.koreamed.org/.

3. BioMed Experts [Internet]. [place unknown]: Elsevier Inc.; [cited 2011 Jun 1]. Available from: http://www.biomedexperts.com/.

4. Scimago Journal \& Country Ranking [Internet]. [place unknown]: Scimago Lab; c2007-2011[cited 2011 Jun 1]. Available from: http://scopus.com/. 